\title{
Long non-coding RNA CASC2 restrains high glucose-induced proliferation, inflammation and fibrosis in human glomerular mesangial cells through mediating miR-135a-5p/TIMP3 axis and JNK signaling
}

Dongju Zhu ${ }^{1 *}$, Xiang Wu² and Qian Xue ${ }^{3}$

\begin{abstract}
Background: Diabetic nephropathy (DN) is a common complication of diabetes. Long non-coding RNA (IncRNA) cancer susceptibility candidate 2 (CASC2) is reported to exert a protective role in DN by a previous study. The working mechanism underlying the protective role of CASC2 in DN progression was further explored in this study.

Methods: The expression of CASC2 and microRNA-135a-5p (miR-135a-5p) was determined by real-time quantitative polymerase chain reaction (RT-qPCR). Cell proliferation ability was assessed by Cell Counting Kit-8 (CCK8) assay and 5-ethynyl-29-deoxyuridine (EDU) assay. Enzyme-linked immunosorbent assay (ELISA) was conducted to analyze the production of inflammatory cytokines in the supernatant. Western blot assay was performed to analyze protein expression. Dual-luciferase reporter assay and RNA immunoprecipitation (RIP) assay were performed to verify the target relationship between miR-135a-5p and CASC2 or tissue inhibitors of metalloproteinase 3 (TIMP3).

Results: High glucose (HG) treatment reduced the expression of CASC2 in human glomerular mesangial cells (HMCs) in a time-dependent manner. CASC2 overexpression suppressed HG-induced proliferation, inflammation and fibrosis in HMCs. miR-135a-5p was validated as a target of CASC2, and CASC2 restrained HG-induced influences in HMCs partly by down-regulating miR-135a-5p. miR-135a-5p bound to the $3^{\prime}$ untranslated region ( $3^{\prime}$ UTR) of TIMP3, and CASC2 positively regulated TIMP3 expression by sponging miR-135a-5p in HMCs. miR-135a-5p silencing inhibited HG-induced effects in HMCs partly by up-regulating its target TIMP3. CASC2 overexpression suppressed HG-induced activation of Jun N-terminal Kinase (JNK) signaling partly through mediating miR-135a-5p/TIMP3 signaling.

Conclusions: In conclusion, CASC2 alleviated proliferation, inflammation and fibrosis in DN cell model by sponging miR-135a-5p to induce TIMP3 expression.
\end{abstract}

Keywords: Diabetic nephropathy, High glucose, CASC2, miR-135a-5p, TIMP3

*Correspondence: wuzidou2020@163.com

1 Department of Nephrology, The Affiliated Hospital, Panzhihua University, Panzhihua 617000, Sichuan, China

Full list of author information is available at the end of the article

(c) The Author(s) 2021. Open Access This article is licensed under a Creative Commons Attribution 4.0 International License, which permits use, sharing, adaptation, distribution and reproduction in any medium or format, as long as you give appropriate credit to the original author(s) and the source, provide a link to the Creative Commons licence, and indicate if changes were made. The images or other third party material in this article are included in the article's Creative Commons licence, unless indicated otherwise in a credit line to the material. If material is not included in the article's Creative Commons licence and your intended use is not permitted by statutory regulation or exceeds the permitted use, you will need to obtain permission directly from the copyright holder. To view a copy of this licence, visit http://creativecommons.org/licenses/by/4.0/. The Creative Commons Public Domain Dedication waiver (http://creativeco mmons.org/publicdomain/zero/1.0/) applies to the data made available in this article, unless otherwise stated in a credit line to the data. 


\section{Background}

Diabetes is a metabolic disease featured by high level of blood sugar over a prolonged period [1]. Diabetic nephropathy (DN) is a serious complication of diabetes, and it is an important inducing factor of cardiovascular disorders and end-stage renal failure [2]. Hyper-proliferation of mesangial cells and deposition of extracellular matrix (ECM) contribute to $\mathrm{DN}$ progression $[3,4]$. Understanding the molecular mechanism behind the aberrant pathological features of mesangial cells is essential to identify novel targets for DN treatment.

Previous study found that long noncoding RNAs (lncRNAs) are widely dysregulated in DN [5]. IncRNAs have been identified as novel targets in the diagnosis, prognosis, and therapy of DN [6]. Accumulating studies reported that lncRNAs regulate the phenotypes of mesangial cells by sponging microRNAs (miRNAs). For instance, $\mathrm{Li}$ et al. found that KCNQ1OT1 silencing alleviates high glucose (HG)-mediated proliferation, oxidative stress, and deposition of ECM in mesangial cells through mediating miR-18b/HMGA2 signaling [7]. Wang et al. demonstrated that IncRNA CTBP1-AS2 attenuates HGmediated oxidative stress, the deposition of ECM, and inflammatory response in mesangial cells by sponging miR-155-5p to induce FOXO1 expression [8]. IncRNA cancer susceptibility candidate 2 (CASC2) is reported to suppress DN progression through different signaling axes $[9,10]$. In this study, the working mechanism of CASC2 in $\mathrm{DN}$ progression was further explored.

miRNAs are implicated in the regulation of cell biological behaviors by binding to the $3^{\prime}$ untranslated region (3'UTR) of target messenger RNAs (mRNAs), causing the translational repression or degradation of mRNAs [11]. Accumulating evidence have uncovered the regulatory roles of miRNAs in the development of diabetes and its associated complications, including DN $[12,13]$. Through bioinformatics prediction, miR-135a-5p was a potential target of CASC2. miR-135a-5p expression is reported to be markedly up-regulated in DN patients relative to that in control group [14]. Zhang et al. demonstrated that miR-135a-5p expression is enhanced in the serum and renal tissue samples of DN patients, and miR-135a-5p absence alleviates transforming growth factor $\beta 1$ (TGF$\beta 1)$-mediated renal fibrosis in DN [15]. In this study, we tested the target relationship between CASC2 and miR$135 a-5 p$ and investigated their functional correlation in DN progression.

Tissue inhibitors of metalloproteinases (TIMPs) are identified as endogenous specific inhibitors of matrix metalloproteinases (MMPs) [16]. The conversion of ECM is modulated by the dynamic balance between the biological activities of MMPs and TIMPs [17]. TIMP3 was predicted as a potential target of miR-135a-5p by bioinformatics database. TIMP3 is the most highly expressed TIMP in kidney, and it is implicated in the regulation of cell inflammatory response and fibrosis [18]. It is reported that TIMP3 expression is reduced in mice with diabetes, and TIMP3 silencing contributes to DN development $[19,20]$. Here, the interaction between miR-135a-5p and TIMP3 was tested, and their functional correlation in DN progression was explored.

HG-induced human glomerular mesangial cells (HMCs) was used as in vitro DN experimental model as previously reported [21-23]. We explored the biological function of lncRNA CASC2 and its associated mechanism in $\mathrm{DN}$ progression using DN cell model.

\section{Materials and methods Cell lines}

Human glomerular mesangial cells (HMCs) and 293T cells were acquired from BeNa Culture Collection (Beijing, China). Cells were cultured in Dulbecco's modified Eagle's medium (DMEM) medium (Gibco, Carlsbad, CA, USA) plus $10 \%$ fetal bovine serum (FBS, Gibco) and $1 \%$ penicillin-streptomycin (Sigma, St. Louis, MO, USA) at $37^{\circ} \mathrm{C}$ with $5 \% \mathrm{CO}_{2}$. HMCs exposed to high glucose (HG; final concentration of $25 \mathrm{mM}$; Sigma) or normal glucose (NG; final concentration of $5.5 \mathrm{mM}$ ) for $48 \mathrm{~h}$ were utilized as DN cell model.

\section{Real-time quantitative polymerase chain reaction (RT-qPCR)}

RNA samples were isolated with Trizol reagent (Invitrogen, Carlsbad, CA, USA). To measure the level of miR135a-5p, reverse transcription was implemented using

Table 1 Specific primers for RT-qPCR

\begin{tabular}{ll}
\hline Gene & Primer sequences $\left(\mathbf{5}^{\prime} \mathbf{3}^{\prime} \mathbf{)}\right.$ \\
\hline CASC2 & Forward primer: AGCCAGAAAATGCATGACACA \\
& Reverse primer: TGGCTCCTCACATCTCCAGT \\
miR-135a-5p & Forward primer: GCCGAGTATGGCTTTTTATT \\
& Reverse primer: GCAGGGTCCGAGGTATTC \\
U6 & Forward primer: GCTTCGGCAGCACATATACTAAAAT \\
& Reverse primer: CGCTTCACGAATTTGCGTGTCAT \\
GAPDH & Forward primer:TATGATGACATCAAGAAGGTGT \\
& Reverse primer:TGTAGCCAAATTCGTTGTCATAC \\
\hline
\end{tabular}


TaqMan microRNA Reverse Transcription kit (Invitrogen), and RT-qPCR was implemented using the specific primers (Table 1) and SYBR mix reagent (Takara, Dalian, China). Complementary DNA (cDNA) of CASC2 was synthesized using High-Capacity cDNA Reverse Transcription kit (Invitrogen) followed by thermal cycle reaction with commercial SYBR mix reagent (Takara). Glyceraldehyde-3-phosphate dehydrogenase (GAPDH) acted as the house-keeping gene for CASC2, whereas U6 acted as the house-keeping gene for miR-135a-5p. The fold changes of gene expression were analyzed by the $2^{-\Delta \Delta \mathrm{Ct}}$ method.

\section{Cell transfection}

The overexpression plasmid of CASC2 (oe-lncRNA CASC2) was constructed by our laboratory, and empty pcDNA $3.1(+)$ vector (vector; Invitrogen) was utilized as the control. The pcDNA $3.1(+)$ vector was digested by Kpn I and Xho I, and the longest transcript of CASC2 (NCBI Reference Sequence: NR_026939.1) was amplified by qPCR and inserted into the vector. miR-135a-5p mimic (5'-UAUGGCUUUUUAUUCCUAUGUG-3'), mimic negative control (mimic NC; 5'-UAGGGCUAG CGUGACGUAAUUG-3), miR-135a-5p inhibitor (5'GUCACACAUGUGACCGUGUUA-3), inhibitor NC (5'-GUUUUGUGCACCAGUGAACA-3), small interfering RNA (siRNA) against TIMP3, including si-TIMP3\#1 (5'-UCCUUUACCAGCUUCUUCCCC-3), si-TIMP3\#2 (5'-AUCUUCAUCUGCUUGAUGGUG-3), and siTIMP3\#3 (5'-ACAUCUUGCCAUCAUAGACGC-3) along with si-NC (5'-UGAUAGACCCGUAAGAUG CAA-3) were purchased from GenePharma (Shanghai, China). When cell confluence reached about $60 \%$, transfection was performed with Lipofectamine 2000 (Invitrogen).

\section{Cell Counting Kit-8 (CCK8) assay}

The optical density was measured to generate proliferation curve to analyze the proliferation capacity of HMCs. HMCs in 96-well plates in the indicated time points were incubated with CCK8 reagent (Dojindo, Tokyo, Japan) for $2 \mathrm{~h}$, and the absorbance $(450 \mathrm{~nm})$ was read using the microplate reader (Invitrogen).

\section{5-ethynyl-29-deoxyuridine (EDU) assay}

HMCs in 24-well plates were incubated with EDU reagent (Sigma) for $2 \mathrm{~h}$. After washing with phosphate buffered saline (PBS) solution (Sangon Biotech, Shanghai, China) twice, cells were immobilized with $4 \%$ paraformaldehyde (EpiZyme, Shanghai, China) and incubated with $0.5 \%$ Triton X-100 (EpiZyme). DAPI dye reagent (EpiZyme) was added to each well to stain the nucleus. Five random fields at the magnification of $200 \times$ were selected. The numbers of EDU ${ }^{+}$cells (proliferative cells) and $\mathrm{DAPI}^{+}$cells (total cells) were analyzed, and the ratio of positive cells was calculated as $\mathrm{EDU}^{+}$cells/DAPI ${ }^{+}$ cells.

\section{Enzyme-linked immunosorbent assay (ELISA)}

The concentrations of monocyte chemotactic protein 1 (Mcp-1), tumor necrosis factor $\alpha$ (TNF- $\alpha$ ), and interleukin 6 (IL-6) in the supernatant were assessed using their matching kits (R\&D Systems, Minneapolis, MN, USA).

\section{Western blot assay}

Total protein samples $(30 \mu \mathrm{g})$ from HMCs were loaded onto the $10 \%$ sodium dodecyl sulfate-polyacrylamide gel electrophoresis (SDS-PAGE), and blotted on polyvinylidene difluoride (PVDF) membrane (Millipore, Billerica, MA, USA). PBS tween-20 (PBST; Sangon Biotech) solution containing $5 \%$ skimmed milk was utilized to block the non-specific sites of the membrane. After that, the membrane was labeled with diluted primary antibodies of proliferating cell nuclear antigen (PCNA; ab18197; 1:8000; Abcam, Cambridge, MA, USA), CyclinD1 (ab16663; 1:10,000; Abcam), TGF- $\beta 1$ (T3176; 1:8000; Sigma), fibronectin (FN; ab2413; 1:5000; Abcam), collagen 4 (Col-4; C1926; 1:5000; Sigma), TIMP3 (ab276134; 1:5000; Abcam), Jun N-terminal Kinase (JNK; ab110724; 1:8000; Abcam), phosphorylated JNK (p-JNK; T183; 1:3000; ab47337) and GAPDH (ab9485; 1:20,000; Abcam). After labeling with horseradish-peroxidase (HRP)-conjugated secondary antibody (1:5000; Abcam), protein bands were visualized using several films and the Super Signal West Pico Chemiluminescent Substrate Kit (Pierce, Rockford, IL, USA). Quantification of protein bands was performed using the Image Lab analysis software (Bio-Rad, Hercules, CA, USA), and the intensities of protein bands were normalized to GAPDH.

\section{Establishment of IncRNA/miRNA/mRNA axis}

LncBase database (http://carolina.imis.athena-innov ation.gr/diana_tools/web/index.php? $\mathrm{r}=\operatorname{lncb}$ asev2/ index-predicted) was utilized to predict the possible miRNA targets of CASC2, and the possible mRNA targets of miR-135a-5p were predicted by StarBase database (http://starbase.sysu.edu.cn).

\section{Dual-luciferase reporter assay}

The target interaction between miR-135a-5p and CASC2 or TIMP3 was verified by dual-luciferase reporter assay. The partial fragment of CASC2 or TIMP3, including the predicted miR-135a-5p binding sites or matching mutant binding sites, was inserted into the downstream of pmirGLO vector (Promega, Madison, WI, USA). The reconstructed luciferase plasmids were termed as lncRNA 
CASC2 wt, lncRNA CASC2 mut, TIMP3 3UTR wt and TIMP3 3UTR mut. These luciferase plasmids were cointroduced with miR-135a-5p mimic or its control into 293T cells. After transfection for 48 h, Firefly luciferase activity and Renilla luciferase activity were determined using the Dual-Luciferase reporter assay system kit (Promega). Renilla luciferase activity was utilized as the control.

\section{RNA immunoprecipitation (RIP) assay}

RIP assay was conducted to extract target-RNA complexes using the Magna RIP RNA-binding Protein Immunoprecipitation kit (Millipore). HMCs were disrupted using the RIP buffer. The antibody against Argonaute 2 (Ago2; Millipore) or immunoglobulin G (IgG; Millipore) was incubated with protein $\mathrm{A} / \mathrm{G}$ beads for $1 \mathrm{~h}$ at $4{ }^{\circ} \mathrm{C}$. The antibody-pre-coated beads were incubated with cell lysates, and the levels of CASC2 and miR-135a-5p were examined by RT-qPCR.

\section{Statistical analysis}

Data analysis was performed using GraphPad Prism 7.0 software (GraphPad, La Jolla, CA, USA). Normally distributed data were expressed as mean \pm standard deviation (SD). The differences between groups were analyzed by unpaired Student's $t$-test (in two groups) or one-way analysis of variance (ANOVA) followed by Tukey's post hoc test (in multiple groups). $P<0.05$ was designated as statistically significant.

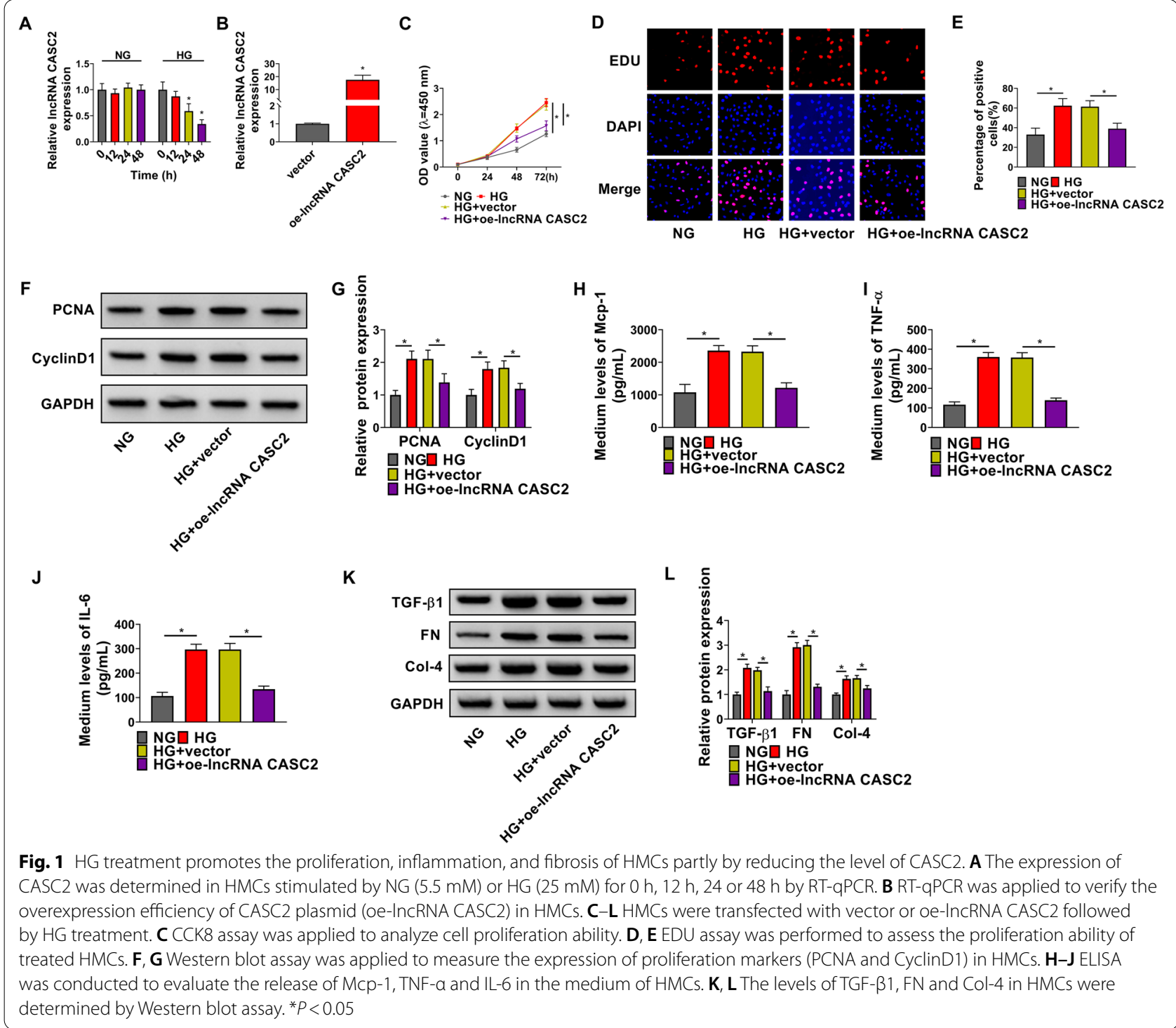




\section{Results}

HG treatment promotes the proliferation, inflammation, and fibrosis of HMCs partly by reducing the level of CASC2 HG (25 mM) treatment decreased the level of CASC2 in HMCs in a time-dependent manner, while NG $(5.5 \mathrm{mM})$ treatment had no significant effect on CASC2 expression in HMCs (Fig. 1A). To test whether the down-regulation of CASC2 was important for HG-induced effects, we rescued the expression of $\mathrm{CASC} 2$ using its overexpression plasmid (oe-lncRNA CASC2) in HG-treated HMCs. The overexpression efficiency of oe-lncRNA CASC2 was high in HMCs (Fig. 1B). CCK8 assay displayed that HG treatment promoted the proliferation of HMCs, which was partly attenuated by the overexpression of CASC2 (Fig. 1C). EDU assay presented that HG exposure promoted the proliferation of HMCs, evidenced by the increased ratio of EDU ${ }^{+}$cells (Fig. 1D, E). Moreover, HG-induced promoting effect on the proliferation of HMCs was attenuated by the addition of CASC2 plasmid (Fig. 1D, E). HG exposure up-regulated the expression of proliferation-associated proteins (PCNA and CyclinD1), and the overexpression of CASC2 reduced the levels of PCNA and CyclinD1 (Fig. 1F, G). Cell inflammatory response was assessed by measuring the release of inflammation-associated cytokines (Mcp-1, TNF- $\alpha$ and
IL-6) via ELISA. HG treatment induced the release of Mcp-1, TNF- $\alpha$ and IL- 6 , and cell inflammatory response was partly attenuated by CASC 2 overexpression in HMCs (Fig. 1H-J). Three fibrosis-associated proteins (TGF- $\beta 1$, FN and Col-4) were detected by Western blot assay to analyze the fibrosis. HG treatment up-regulated the expression of fibrosis-associated proteins (TGF- $\beta 1$, FN and Col-4), which was partly alleviated by the overexpression of CASC2 in HMCs (Fig. 1K, L). These data suggested that HG-induced pro-proliferative, pro-inflammatory and pro-fibrotic effects were partly dependent on the down-regulation of CASC2.

\section{miR-135a-5p is a target of CASC2}

We predicted the potential miRNA targets of CASC2 using LncBase database, and miR-135a-5p was a candidate target of CASC2 on the basis of their complementary sites (Fig. 2A). We cloned the fragment of CASC2, containing the wild-type or the mutant type binding sites with miR-135a-5p, into the downstream of Firefly luciferase gene to obtain luciferase reporter plasmid lncRNA CASC2 wt or lncRNA CASC2 mut (Fig. 2B). The luciferase activity of wild-type plasmid (lncRNA CASC2 wt) was markedly reduced by the overexpression of miR135a-5p, while the luciferase activity of mutant plasmid

\section{A \\ IncRNA CASC2 wt 5'-AGCAUUAAAGUUAUUUUUACAAAGCCAUC-3' miR-135a-5p 3'-GUGUA-----UCCUUAUU------UUUCGGUAU-5'} IncRNA CASC2 mut 5'-AGCAUUAAUGUUAUUUUUACUUUCGGUAC-3'

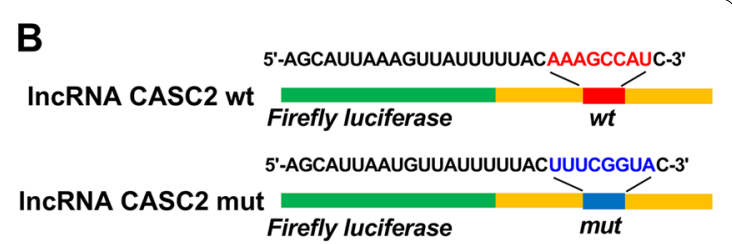

E

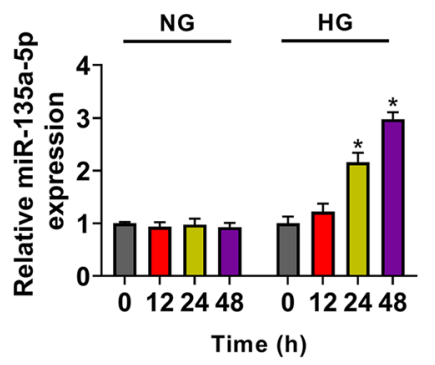

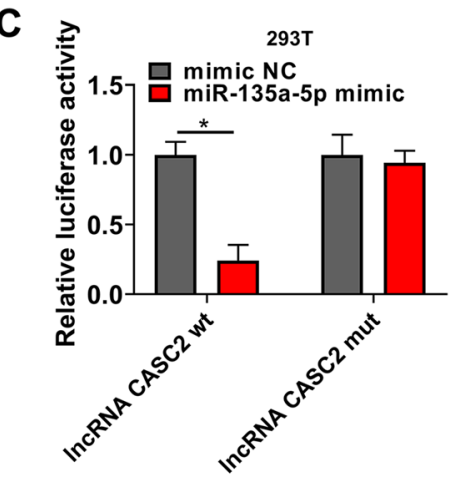

D

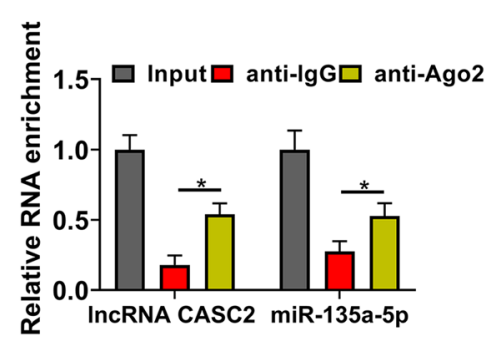


(lncRNA CASC2 mut) was unchanged by the addition of miR-135a-5p or mimic NC (Fig. 2C), suggesting the interaction between CASC2 and miR-135a-5p in 293T cells. RIP assay was conducted to verify the target relationship between CASC2 and miR-135a-5p in HMCs. When using Ago2 antibody, both CASC2 and miR135a-5p were enriched (Fig. 2D), demonstrating the target interaction between CASC2 and miR-135a-5p in RNA-induced silencing complex (RISC). HG treatment time-dependently increased the expression of miR135a-5p in HMCs (Fig. 2E). These results revealed that miR-135a-5p was a target of CASC2 in HMCs.

\section{CASC2 overexpression restrains the proliferation,} inflammation and fibrosis of HG-induced HMCs partly by down-regulating its target miR-135a-5p

The overexpression efficiency of miR-135a-5p mimic was high in HMCs (Fig. 3A). CASC2 overexpression reduced the level of miR-135a-5p (Fig. 3B), suggesting the negative regulatory relationship between CASC2 and miR135a-5p in HMCs. To explore whether CASC2 exerted its function by targeting miR-135a-5p, we performed rescue experiments. The addition of miR-135a-5p mimic largely rescued the level of miR-135a-5p in CASC2overexpressed HMCs (Fig. 3B). Through CCK8 assay and EDU assay, we found that CASC2 overexpressionmediated suppressive effect on cell proliferation was largely counteracted by the addition of miR-135a-5p mimic (Fig. 3C-E). CASC2 overexpression-mediated suppressive effects on the levels of pro-proliferation proteins (PCNA and CyclinD1) were largely counteracted by the overexpression of miR-135a-5p (Fig. 3F, G). miR$135 \mathrm{a}-5 \mathrm{p}$ overexpression largely rescued the production of inflammatory cytokines in CASC2-overexpressed HMCs upon $\mathrm{HG}$ treatment (Fig. $3 \mathrm{H}-\mathrm{J}$ ). The expression of fibrosis-associated proteins (TGF- $\beta 1, \mathrm{FN}$ and Col-4) was down-regulated in CASC2-overexpressed HMCs upon HG treatment, and the accumulation of miR-135a-5p largely rescued the levels of TGF- $\beta 1$, FN and Col-4 (Fig. 3K, L). These data suggested that CASC2 overexpression suppressed HG-induced proliferation, inflammation and fibrosis of HMCs partly by down-regulating miR-135a-5p.

\section{miR-135a-5p binds to the 3'UTR of TIMP3}

The mRNA targets of miR-135a-5p were predicted by StarBase database, and the putative binding sites between miR-135a-5p and TIMP3 were shown in Fig. 4A. We constructed luciferase reporter plasmid named TIMP3 3'UTR wt or TIMP3 3'UTR mut that contained the wildtype or the mutant type predicted binding sites with miR-135a-5p (Fig. 4B). Luciferase activity was dramatically reduced in 293T cells in TIMP3 3UTR wt group when co-transfected with miR-135a-5p mimic rather than mimic NC (Fig. 4C), suggesting the target interaction between miR-135a-5p and TIMP3. When the putative binding sites in TIMP3 were mutated, the luciferase activity was no longer reduced by the overexpression of miR-135a-5p (Fig. 4C), suggesting that miR-135a-5p bound to TIMP3 via the putative sites. HG treatment decreased the protein level of TIMP3 in a time-dependent manner in HMCs (Fig. 4D, E). CASC2 overexpression up-regulated the protein expression of TIMP3, which was partly attenuated by the accumulation of miR-135a-5p in HMCs (Fig. 4F), suggesting that CASC2 up-regulated TIMP3 expression partly by sponging miR-135a-5p. Overall, TIMP3 was confirmed as a target of miR-135a-5p, and it was regulated by CASC2/miR$135 \mathrm{a}-5 \mathrm{p}$ signaling.

\section{miR-135a-5p knockdown suppresses HG-induced effects in HMCs partly by up-regulating TIMP3}

We performed rescue experiments through transfecting HMCs with miR-135a-5p inhibitor alone or together with the siRNA of TIMP3 (si-TIMP3\#1, si-TIMP3\#2, or si-TIMP3\#3). The silencing efficiency of miR-135a-5p inhibitor was high in HMCs (Fig. 5A). Also, Western blot assay confirmed the high interference efficiencies of si-TIMP3\#1, si-TIMP3\#2, and si-TIMP3\#3 in HMCs (Fig. 5B). Transfection with miR-135a-5p inhibitor significantly up-regulated the protein expression of TIMP3, whereas the addition of TIMP3 siRNA reduced the expression of TIMP3 again (Fig. 5C). miR-135a-5p interference suppressed the proliferation in HG-treated HMCs (Fig. 5D, F). The results of Western blot assay also manifested that miR-135a-5p knockdown downregulated the levels of PCNA and CyclinD1 in HGtreated HMCs (Fig. 5G, H). In addition, we found that the knockdown of miR-135a-5p restrained the inflammatory response (Fig. 5I, K) and fibrosis (Fig. 5 L, M) of HG-induced HMCs. With the interference of TIMP3, cell proliferation ability was largely recovered on the basis of the results of CCK8 assay, EDU assay and Western blot assay (Fig. 5D, H). TIMP3 knockdown also largely rescued the inflammation and fibrosis in miR-135a-5psilenced HMCs upon HG treatment (Fig. 5I, M). These results indicated that miR-135a-5p knockdown attenuated HG-induced proliferation, inflammation and fibrosis of HMCs partly through enhancing the level of TIMP3.

\section{HG treatment increases the phosphorylation level of JNK partly by targeting CASC2/miR-135a-5p/TIMP3 axis}

JNK signaling is identified to be aberrantly activated in DN patients, and suppressing the activation of JNK signaling is a novel treatment strategy for DN patients $[24,25]$. HG treatment had no effect in the total protein 


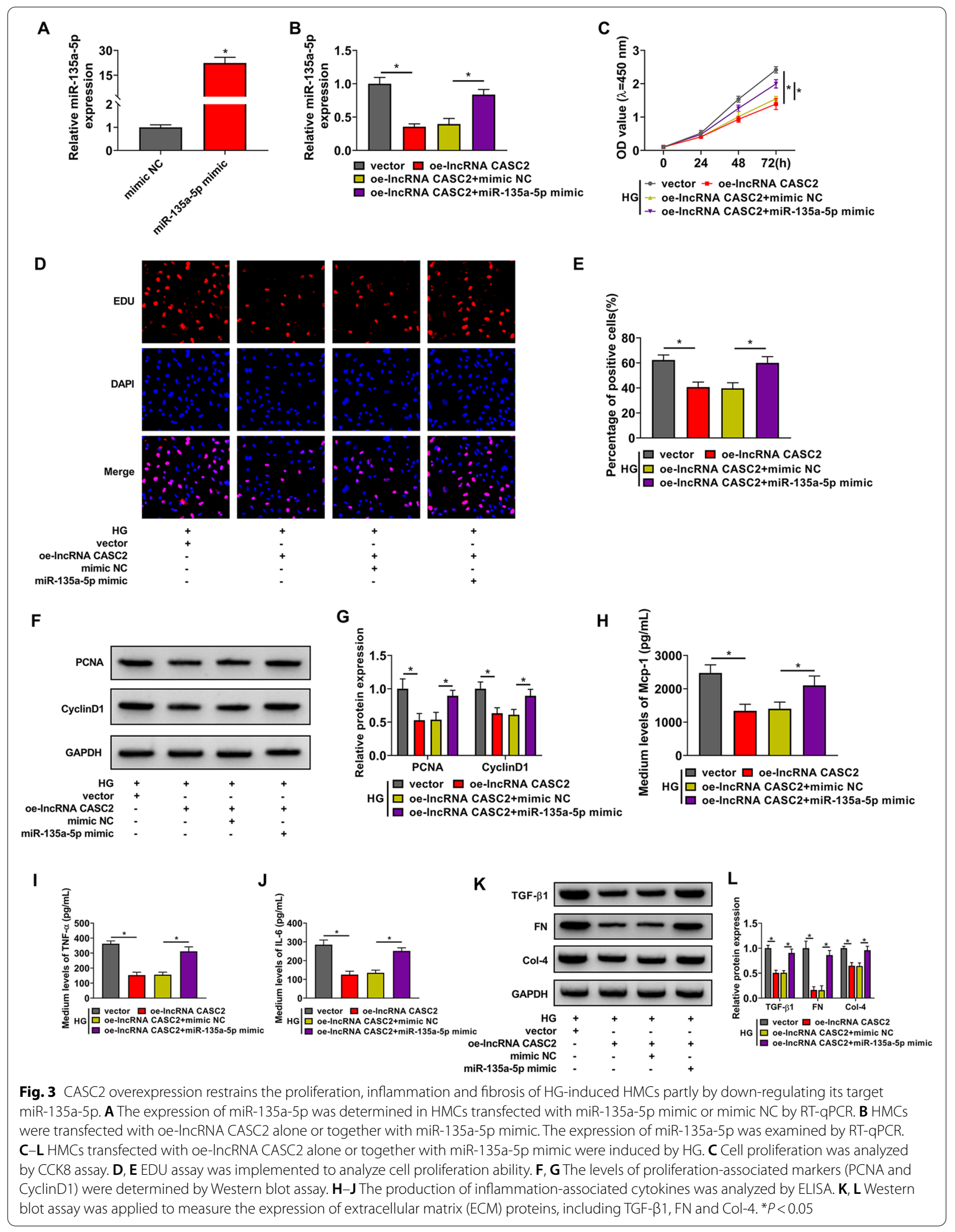




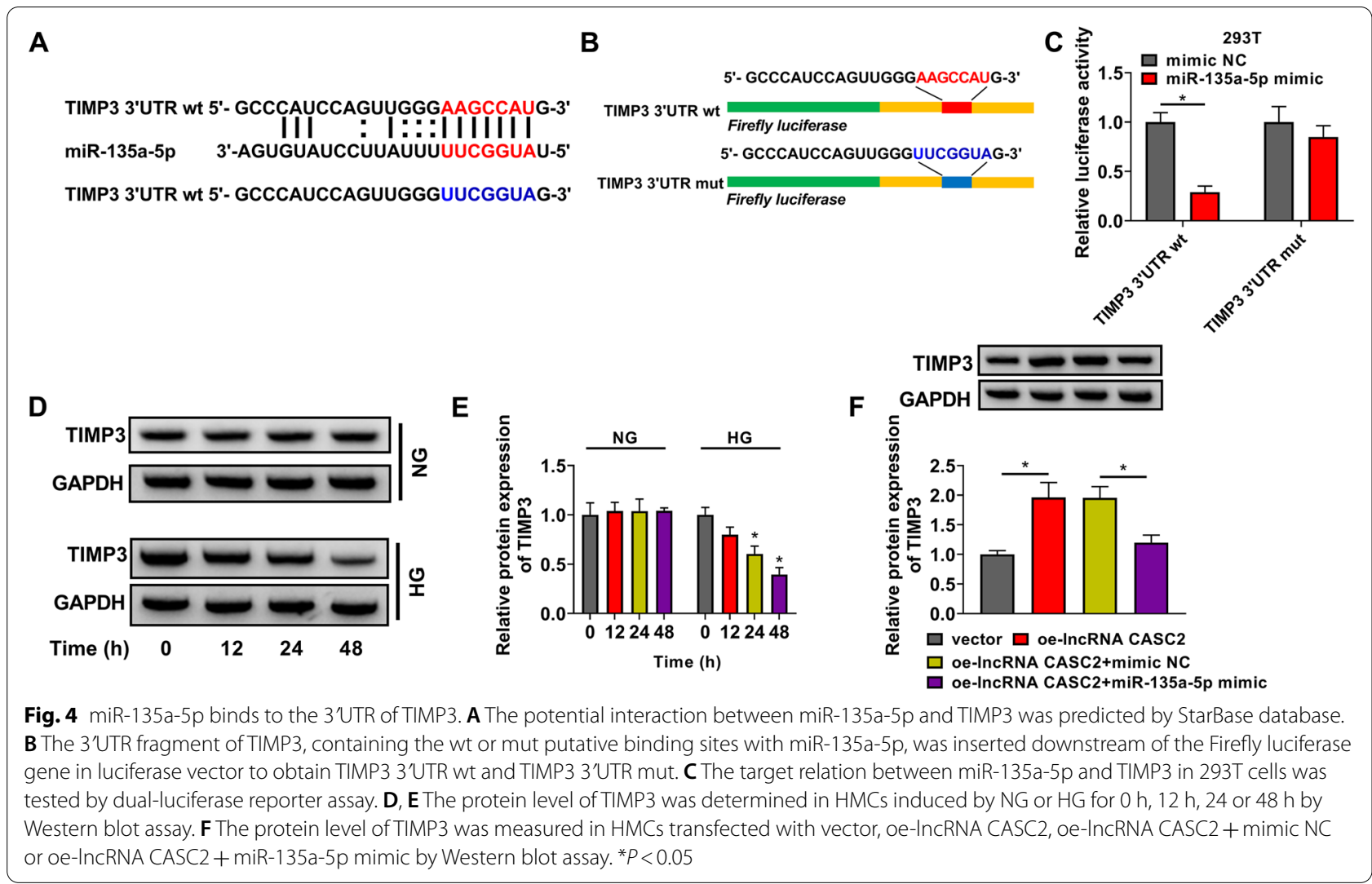

level of JNK, but markedly increased the phosphorylation level of JNK in HMCs (Fig. 6A, B). The overexpression of CASC2 suppressed HG-induced phosphorylation of JNK (Fig. 6A, B). The overexpression of miR-135a-5p or the silence of TIMP3 partly rescued the phosphorylation level of JNK in CASC2-overexpressed HMCs upon HG treatment (Fig. 6C, D). Moreover, the results in Additional file 1: Figure S1 presented that the effect of CASC2 overexpression was slightly weaker than the effect of JNK1/2 inhibitor SP600125 in inactivating JNK signaling in HG-induced HMCs, suggesting that CASC2 plays a role similar to the JNK1/2 inhibitor SP600125 in HGinduced HMCs. These data demonstrated that CASC2 overexpression inactivated JNK signaling partly through targeting miR-135a-5p/TIMP3 axis in HG-induced HMCs.

\section{Discussion}

Accumulating evidence have demonstrated that lncRNAs play important regulatory roles in the progression of DN $[26,27]$. For instance, lncRNA XIST is reported to alleviate HG-induced damage in podocyte in DN through mediating miR-30/AVEN signaling cascade [28]. Ji et al. reported that the silence of IncRNA MIAT attenuates HG-induced proliferation and fibrosis in mesangial cells by down-regulating E2F3 level [29]. Li et al. found that lncRNA KCNQ1OT1 interference alleviates HG-induced proliferation, oxidative stress and deposition of ECM in HMCs through regulating miR-18b/HMGA2 signaling [7]. As for IncRNA CASC2, Min et al. found that CASC2 attenuates DN development by targeting miR$144 /$ SOCS2 axis [9]. Zhang et al. found that CASC2 is down-regulated in the serum samples of DN patients and HG-treated HMCs, and CASC2 inhibits HG-mediated proliferation, oxidative stress and the accumulation of ECM in HMCs by regulating miR-133b/FOXP1 signaling [10]. We built DN cell model through exposing HMCs to HG. We found that HG time-dependently reduced the expression of CASC2 in HMCs. Furthermore, CASC2 overexpression alleviated HG-induced proliferation, inflammatory response and fibrosis of HMCs. These results suggested that $\mathrm{CASC} 2$ was a potential target for the intervention of $\mathrm{DN}$ progression.

Subsequently, we intended to investigate the molecular mechanism behind the biological function of CASC2 in DN. Accumulating studies have shown that lncRNAs exert their biological roles by acting as miRNA sponges $[30,31]$. For example, lncRNA SNHG8 is reported to contribute to the development of prostate cancer by elevating HOXB7 expression via sponging miR-384 
(See figure on next page.)

Fig. 5 miR-135a-5p knockdown suppresses HG-induced effects in HMCs partly by up-regulating TIMP3. A The level of miR-135a-5p in HMCs transfected with inhibitor NC or miR-135a-5p inhibitor was determined by RT-qPCR. B The silencing efficiency of si-TIMP3 in HMCs was analyzed by Western blot assay. C HMCs were transfected with inhibitor NC, miR-135a-5p inhibitor, miR-135a-5p inhibitor + si-NC or miR-135a-5p inhibitor + si-TIMP3. Western blot assay was employed to assess the protein expression of TIMP3 in transfected HMCs. D-M HMCs transfected with inhibitor NC, miR-135a-5p inhibitor, miR-135a-5p inhibitor + si-NC or miR-135a-5p inhibitor + si-TIMP3 were induced by HG. D-F CCK8 assay and EDU assay were utilized to analyze cell proliferation ability. G, H Western blot assay was employed to detect the levels of PCNA and CyclinD1 in HMCs. I-K The levels of inflammatory cytokines (Mcp-1, TNF- $a$ and IL-6) were analyzed by ELISA. L, $\mathbf{M}$ Western blot assay was utilized to analyze the expression of fibrosis-associated proteins in HMCs. ${ }^{*} P<0.05$

[32]. IncRNA MCM3AP-AS1 is reported to restrain the development of colorectal cancer through mediating miR-19a-3p/FOXF2 signaling [33]. We predicted the possible miRNA targets of CASC2 using LncBase database, and miR-135a-5p was confirmed as a target of CASC2 by dual-luciferase reporter assay and RIP assay. He et al. found that miR-135a is significantly up-regulated in DN patients, and miR-135a accelerates the renal fibrosis by targeting TRPC1 in DN [14]. Peng et al. demonstrated that circular RNA_010383 silencing facilitates DN progression by functioning as a sponge for miR-135a [34]. Zhang et al. claimed that miR-135a-5p level is enhanced in the serum and renal tissues of $\mathrm{DN}$ patients, and miR$135 \mathrm{a}-5 \mathrm{p}$ interference suppresses DN progression by regulating SIRT1 [35]. Consistent with these articles, we found that HG up-regulated the expression of miR$135 a-5 p$ in a time-dependent manner in HMCs. CASC2 overexpression-mediated protective effects in HGinduced HMCs were largely overturned by the addition of miR-135a-5p mimic, suggesting that CASC2 overexpression attenuated HG-induced effects in HMCs largely by down-regulating miR-135a-5p.

To further explore the working mechanism of miR$135 \mathrm{a}-5 \mathrm{p}$ in DN progression, the downstream targets of miR-135a-5p were predicted using StarBase database. TIMP3 was verified as a target of miR-135a-5p. TIMP3 is reported to exert important function in maintaining kidney homeostasis, and the down-regulation of TIMP3 is identified as a marker for DN [20]. Chen et al. found that miR-21 contributes to DN progression through reducing the expression of TIMP3 [36]. Wang et al. found that lncRNA TUG1 inhibits DN progression by sponging miR-21 to induce the expression of TIMP3 [37]. These results confirmed the protective role of TIMP3 in DN. We found that HG down-regulated TIMP3 level in a time-dependent manner in HMCs. CASC2 can positively regulate TIMP3 expression by acting as miR-135a-5p sponge in HMCs. Compensation experiments revealed that miR-135a-5p silencing attenuated HG-induced effects in HMCs partly by upregulating TIMP3.

The abnormal activation of JNK signaling contributes to DN progression [24], and JNK signaling has been regarded as a therapeutic target for DN patients [25]. We found that HG treatment elevated the phosphorylation level of JNK in HMCs, and CASCs overexpression suppressed HG-induced phosphorylation of JNK partly through targeting miR-135a-5p/TIMP3 signaling.

However, there were several limitations of our study that should be noted. There were no clinical data about the expression of CASC2/miR-135a-5p/TIMP3 axis in DN patients and healthy volunteers, and only one cell line was chosen in this study. Furthermore, in vivo experiments were lacking in this study. Therefore, the results may be unpersuasive to some extent. In future, more study needs to be conducted to verify the results in this study.

In conclusion, HG treatment up-regulated the expression of miR-135a-5p and reduced the levels of CASC2 and TIMP3 in HMCs. CASC2 protected HMCs from HG-induced proliferation, inflammation and fibrosis largely through targeting miR-135a-5p/TIMP3 signaling (Fig. 7).

\section{Abbreviations}

DN: Diabetic nephropathy; IncRNA: Long non-coding RNA; CASC2: Cancer susceptibility candidate 2; miR-135a-5p: microRNA-135a-5p; RT-qPCR: Realtime quantitative polymerase chain reaction; CCK8: Cell Counting Kit-8; ELISA: enzyme-linked immunosorbent assay.

\section{Supplementary Information}

The online version contains supplementary material available at https://doi. org/10.1186/s13098-021-00709-5.

Additional file 1: Figure S1. CASC2 plays a role similar to the JNK1/2 inhibitor SP600125 in HG-induced HMCs. The levels of JNK and p-JNK were detected in HMCs in the following five groups by Western blot assay: $\mathrm{NG}, \mathrm{HG}, \mathrm{HG}$ + vector, HG + oe-IncRNA CASC2, and HG + SP600125 (20 $\mu \mathrm{M} / 24 \mathrm{~h}) .{ }^{*} P<0.05$.

\section{Acknowledgements \\ None.}

\section{Authors' contributions}

DZ designed the research and wrote the manuscript. XW collected the data. QX analyzed the data and performed the statistical analysis. DZ, XW and QX reviewed and edited the manuscript. All authors read and approved the final manuscript. 


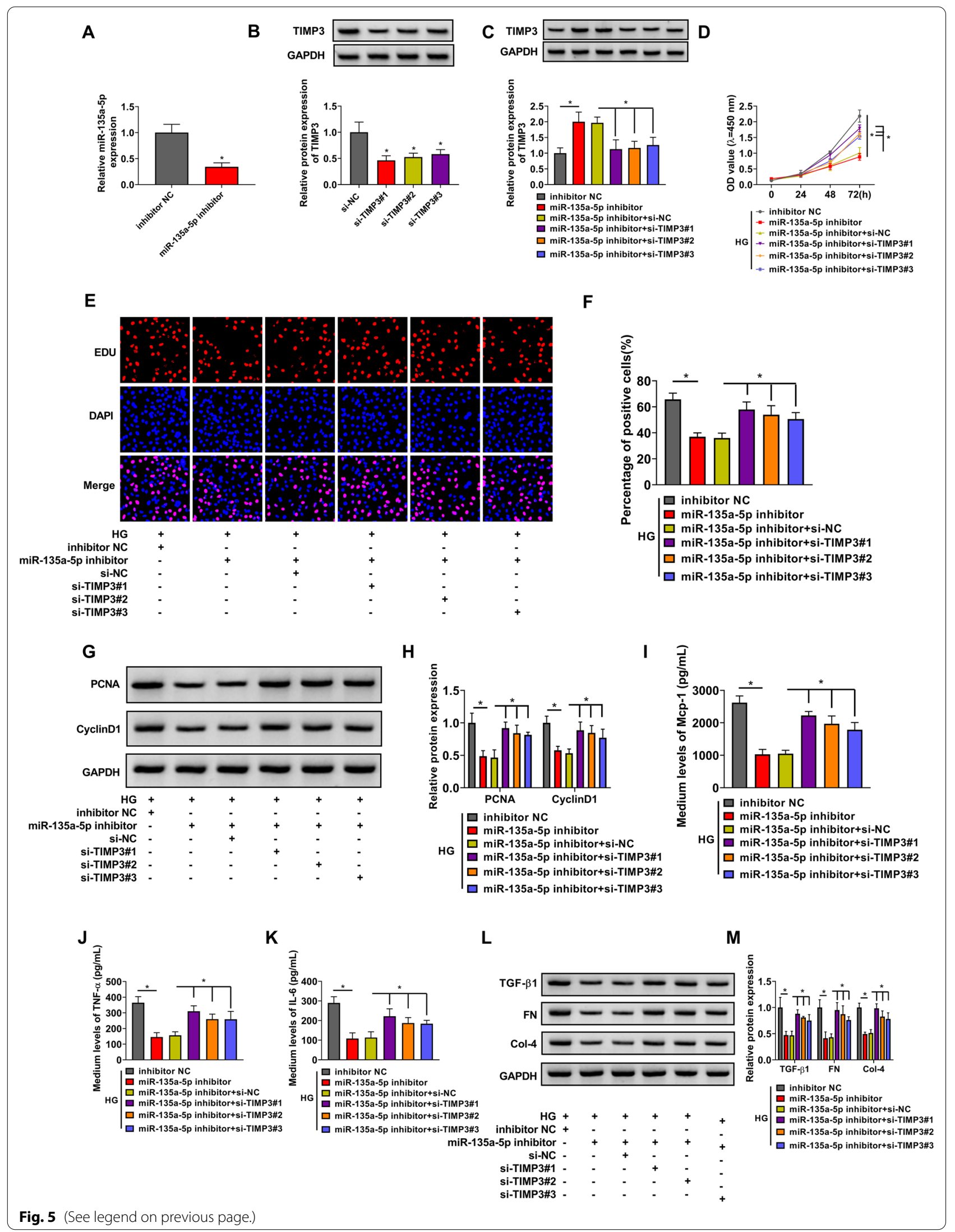



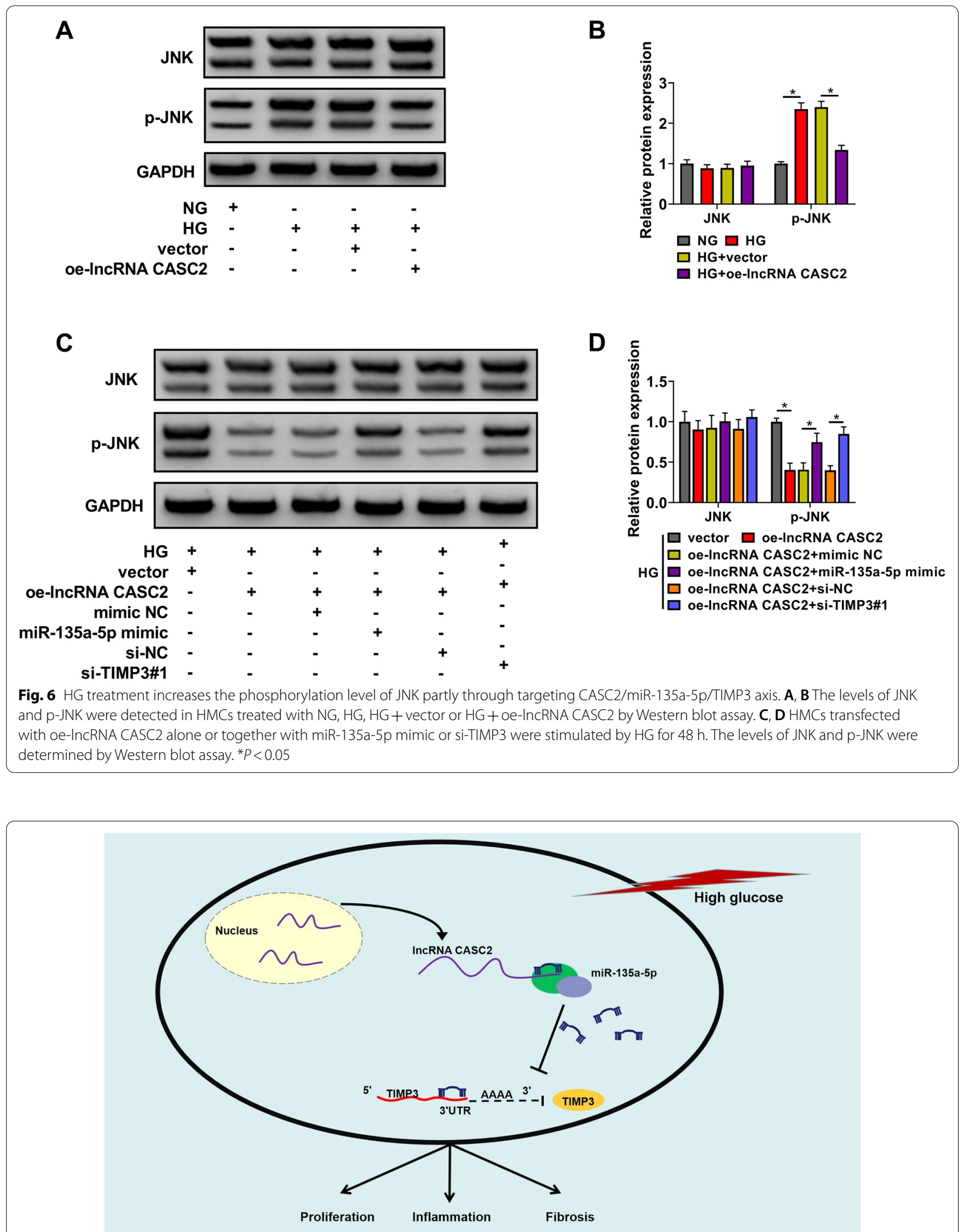

Fig. 7 HG treatment promotes the proliferation, inflammation and fibrosis of HMCs partly through regulating CASC2/miR-135a-5p/TIMP3 signaling 


\section{Funding \\ None.}

\section{Availability of data and materials}

Not applicable.

\section{Declarations}

\section{Ethics approval and consent to participate}

Not applicable.

\section{Consent for publication}

All authors read and approved the final manuscript.

\section{Competing interests}

The authors declare that they have no conflicts of interest.

\section{Author details}

'Department of Nephrology, The Affiliated Hospital, Panzhihua University, Panzhihua 617000, Sichuan, China. ${ }^{2}$ Department of Pediatrics, Panzhihua Central Hospital, Panzhihua 617000, Sichuan, China. ${ }^{3}$ Department of Gas troenterology, The First Affiliated Hospital of Chongqing Medical University, Chongqing 400000, China.

\section{Received: 16 March 2021 Accepted: 12 August 2021}

Published online: 26 August 2021

\section{References}

1. Rubino F. Medical research: time to think differently about diabetes. Nature. 2016;533(7604):459-61.

2. Lei L, Mao Y, Meng D, Zhang X, Cui L, Huo Y, Wang Y. Percentage of circulating CD8+ Tlymphocytes is associated with albuminuria in type 2 diabetes mellitus. Exp Clin Endocrinol Diabetes. 2014;122(1):27-30.

3. Cove-Smith A, Hendry BM. The regulation of mesangial cell proliferation. Nephron Exp Nephrol. 2008;108(4):e74-9.

4. Kolset SO, Reinholt FP, Jenssen T. Diabetic nephropathy and extracellular matrix. J Histochem Cytochem. 2012;60(12):976-86.

5. Hao S, Cong L, Qu R, Liu R, Zhang G, Li Y. Emerging roles of circular RNAs in colorectal cancer. OncoTargets Ther. 2019;12:4765-77.

6. Guo J, Liu Z, Gong R. Long noncoding RNA: an emerging player in diabetes and diabetic kidney disease. Clin Sci (Lond). 2019;133(12):1321-39.

7. Li Y, Zang H, Zhang X, Huang G. circ_0136666 facilitates the progression of colorectal cancer via miR-383/CREB1 axis. Cancer Manag Res. 2020:12:6795-806.

8. Xu D, Wu Y, Wang X, Hu X, Qin W, Li Y, Wang Y, Zhang Z, Lu S, Sun T, et al. Identification of functional circRNA/miRNA/mRNA regulatory network for exploring prospective therapy strategy of colorectal cancer. J Cell Biochem. 2020;121:4785-97.

9. Min XQ, Xie Y. IncRNA CASC2 alleviates the progression of diabetic nephropathy by regulating the miR-144/SOCS2 signalling axis. Kidney Blood Press Res. 2020:45(6):837-49.

10. Zhang $X L$, Zhu HQ, Zhang Y, Zhang CY, Jiao JS, Xing XY. IncRNA CASC2 regulates high glucose-induced proliferation, extracellular matrix accumulation and oxidative stress of human mesangial cells via miR-133b/ FOXP1 axis. Eur Rev Med Pharmacol Sci. 2020;24(2):802-12.

11. He L, Hannon GJ. MicroRNAs: small RNAs with a big role in gene regulation. Nat Rev Genet. 2004;5(7):522-31.

12. Lu Z, Liu N, Wang F. Epigenetic regulations in diabetic nephropathy. J Diabetes Res. 2017;2017:7805058.

13. Cui C, Cui Y, Fu Y, Ma S, Zhang S. Microarray analysis reveals gene and microRNA signatures in diabetic kidney disease. Mol Med Rep. 2018;17(2):2161-8.

14. He F, Peng F, Xia X, Zhao C, Luo Q, Guan W, Li Z, Yu X, Huang F. MiR-135a promotes renal fibrosis in diabetic nephropathy by regulating TRPC1. Diabetologia. 2014;57(8):1726-36.

15. Zhang L, Dong X, Yan B, Yu W, Shan L. CircAGFG1 drives metastasis and stemness in colorectal cancer by modulating YY1/CTNNB1. Cell Death Dis. 2020;11(7):542.
16. Wei S, Xie Z, Filenova E, Brew K. Drosophila TIMP is a potent inhibitor of MMPs and TACE: similarities in structure and function to TIMP-3. Biochemistry. 2003;42(42):12200-7.

17. Xu X, Xiao L, Xiao P, Yang S, Chen G, Liu F, Kanwar YS, Sun L. A glimpse of matrix metalloproteinases in diabetic nephropathy. Curr Med Chem. 2014;21(28):3244-60

18. Kassiri Z, Oudit GY, Kandalam V, Awad A, Wang X, Ziou X, Maeda N, Herzenberg AM, Scholey JW. Loss of TIMP3 enhances interstitial nephritis and fibrosis. J Am Soc Nephrol. 2009;20(6):1223-35.

19. Basu R, Lee J, Wang Z, Patel VB, Fan D, Das SK, Liu GC, John R, Scholey JW, Oudit GY, et al. Loss of TIMP3 selectively exacerbates diabetic nephropathy. Am J Physiol Renal Physiol. 2012;303(9):F1341-52.

20. Fiorentino L, Cavalera M, Menini S, Marchetti V, Mavilio M, Fabrizi M, Conserva F, Casagrande V, Menghini R, Pontrelli P, et al. Loss of TIMP3 underlies diabetic nephropathy via FoxO1/STAT1 interplay. EMBO Mol Med. 2013:5(3):441-55.

21. Zhang X, Shang J, Wang X, Cheng G, Jiang Y, Liu D, Xiao J, Zhao Z. Microarray analysis reveals long non-coding RNA SOX2OT as a novel candidate regulator in diabetic nephropathy. Mol Med Rep. 2018;18(6):5058-68.

22. Li Y, Zheng LL, Huang DG, Cao H, Gao YH, Fan ZC. LNCRNA CDKN2B-AS1 regulates mesangial cell proliferation and extracellular matrix accumulation via miR-424-5p/HMGA2 axis. Biomed Pharmacother Biomed Pharmacother. 2020;121:109622.

23. Wang G, Yan Y, Xu N, Hui Y, Yin D. Upregulation of microRNA-424 relieved diabetic nephropathy by targeting Rictor through mTOR Complex2/Protein Kinase B signaling. J Cell physiol. 2019;234(7):11646-53.

24. Lian H, Cheng Y, Wu X. TMEM16A exacerbates renal injury by activating P38/JNK signaling pathway to promote podocyte apoptosis in diabetic nephropathy mice. Biochem Biophys Res Commun. 2017;487(2):201-8.

25. Wang Y, Wang Y, Luo M, Wu H, Kong L, Xin Y, Cui W, Zhao Y, Wang J, Liang $\mathrm{G}$, et al. Novel curcumin analog C66 prevents diabetic nephropathy via JNK pathway with the involvement of p300/CBP-mediated histone acetylation. Biochim Biophys Acta. 2015;1852(1):34-46.

26. Ignarski M, Islam R, Muller RU. Long non-coding RNAs in kidney disease. Int J Mol Sci. 2019;20(13):3276.

27. Leti F, Morrison E, DiStefano JK. Long noncoding RNAs in the pathogenesis of diabetic kidney disease: implications for novel therapeutic strategies. Per Med. 2017;14(3):271-8.

28. Long B, Wan Y, Zhang S, LV L. InCRNA XIST protects podocyte from high glucose-induced cell injury in diabetic nephropathy by sponging miR-30 and regulating AVEN expression. Arch Physiol Biochem. 2020. https://doi. org/10.1080/13813455.2020.1854307.

29. Ji TT, Qi YH, Li XY, Tang B, Wang YK, Zheng PX, Li W, Qu X, Feng L, Bai SJ. Loss of InCRNA MIAT ameliorates proliferation and fibrosis of diabetic nephropathy through reducing E2F3 expression. J Cell Mol Med. 2020;24:13314-23.

30. Thomson DW, Dinger ME. Endogenous microRNA sponges: evidence and controversy. Nat Rev Genet. 2016; 17(5):272-83.

31. Bayoumi AS, Sayed A, Broskova Z, Teoh JP, Wilson J, Su H, Tang YL, Kim IM. Crosstalk between Long Noncoding RNAs and MicroRNAs in Health and Disease. Int J Mol Sci. 2016;17(3):356.

32. Shi Z, Zhang H, Jie S, Yang $X$, Huang $Q$, Mao Y, Zhang Y. Long non-coding RNA SNHG8 promotes prostate cancer progression through repressing miR-384 and up-regulating HOXB7. J Gene Med. 2021;23:e3309.

33. Dai W, Zeng W, Lee D. InCRNA MCM3AP-AS1 inhibits the progression of colorectal cancer via the miR-19a-3p/FOXF2 axis. J Gene Med. 2021;23:e3306

34. Peng F, Gong W, Li S, Yin B, Zhao C, Liu W, Chen X, Luo C, Huang Q, Chen T, et al. circRNA_010383 acts as a sponge for miR-135a and its downregulated expression contributes to renal fibrosis in diabetic nephropathy. Diabetes. 2020;70:603-15

35. Zhang J, Zhang L, Zha D, Wu X. Inhibition of miRNA135a5p ameliorates TGFbeta1induced human renal fibrosis by targeting SIRT1 in diabetic nephropathy. Int J Mol Med. 2020;46(3):1063-73.

36. Chen $X$, Zhao $L$, Xing $Y$, Lin B. Down-regulation of microRNA-21 reduces inflammation and podocyte apoptosis in diabetic nephropathy by relieving the repression of TIMP3 expression. Biomed Pharmacother. 2018;108:7-14

37. Wang F, Gao X, Zhang R, Zhao P, Sun Y, Li C. IncRNA TUG1 ameliorates diabetic nephropathy by inhibiting miR-21 to promote TIMP3-expression. Int J Clin Exp Pathol. 2019;12(3):717-29. 
Publisher's Note

Springer Nature remains neutral with regard to jurisdictional claims in published maps and institutional affiliations.
Ready to submit your research? Choose BMC and benefit from:

- fast, convenient online submission

- thorough peer review by experienced researchers in your field

- rapid publication on acceptance

- support for research data, including large and complex data types

- gold Open Access which fosters wider collaboration and increased citations

- maximum visibility for your research: over 100M website views per year

At BMC, research is always in progress.

Learn more biomedcentral.com/submissions 